\title{
Discovering Method of Control of the "Dzhanibekov's Effect" and Proposing its Applications for the Possible Future Space Missions
}

\author{
By Pavel M. TrivaILO ${ }^{1)}$ and Hirohisa KoJIMA ${ }^{2)}$ \\ ${ }^{1)}$ School of Engineering, RMIT University, Melbourne, Australia \\ ${ }^{2)}$ Department of Aerospace Engineering, Tokyo Metropolitan University, Hino, Japan
}

(Received June 30th, 2017)

\begin{abstract}
This paper is dedicated to the numerical simulation and analysis of the "Dzhanibekov's Effect" - non-stable "flipping" motion of the rigid body with periodic change by $180^{\circ}$ of the direction of the main axis of its rotation, always occurring when the body is provided with the main rotation about its axis with intermediate principal moment of inertia. In this work we are proposing and developing a new concept of "inertial morphing" of the spacecraft for the versatile control of the "Dzhanibekov's Effect", enabling manipulations with the attitude of the spacecraft without employing classical gyroscopes. We are demonstrating that applying controlled changes to the inertial properties of the system, the "Dzhanibekov's Effect" flipping motion can be completely stopped, if this motion is undesirable. Similarly, the "Dzhanibekov's Effect" flipping motion can be activated on the stable, non-tumbling spacecraft, if this is desired for the purposes of the mission. We are also showing that the frequency of the flipping motion can be controlled within a wide range. For the implementation of the transformations between stable and unstable modes of motion, we are proposing two main conceptual solutions, involving changes to the system, resulting in the intermediate moment of inertia becoming the smallest or largest principal moment of inertia of the body. Development of the conceptual 6-mass model of the spacecraft enabling controllable switching OFF of the "Dzhanibekov's Effect" flipping is presented. Furthermore, we also exploring the possibilities of utilization of the "Dzhanibekov's Effect" for possible future new space missions, employing periodic change in the attitude orientation of the spacecraft. It is believed, that new results of this research may have multiple applications for possible future space missions. In particular, we are suggesting assistance in establishing formation flight; thruster direction control and control of the period of the "flipping" motion of the spacecraft.
\end{abstract}

Key Words: "Dzhanibekov's Effect”, Rigid Body Dynamics, Euler’s Equations of Motion, Morphing Spacecraft, Tumbling Motion

\section{Nomenclature}

$\begin{array}{cc}d & : \\ \mathcal{G} & : \\ \overrightarrow{\mathbf{H}}(t) & : \\ H & : \\ I_{x x}, I_{y y}, I_{z z} & : \\ \mathcal{K}_{0} & : \\ K(k) & : \\ k & : \\ \mathcal{M} & :\end{array}$

$M a a, M b b, M c c$ :

$m_{x}, m_{y}, m_{z}$

$\overrightarrow{\mathbf{N}}(t)$

$N_{x}, N_{y}, N_{z}$

$\mathcal{P}$

$P, Q, R$

$r_{x}, r_{y}, r_{z}$

$T$

$t$

$x, y, z$

$\mathbf{x}$

$\psi, \theta, \phi$

$\vec{\omega}(t)$

$\omega_{x}, \omega_{y}, \omega_{z}$

Subscripts

$f$ derivative

mass center of the rigid body/spacecraft angular momentum vector magnitude of the angular momentum vector principal moments of inertia initial kinetic energy of the system complete elliptic integral of the 1 st kind parameter in complete elliptic integral $K$ mass matrix moments of inertia in original Euler's work dumbbell masses in the 6-mass spacecraft torque vector torque components pivot point torque components in original Euler's work axial positions of the spacecraft masses period of the unstable "flipping" motion time principal axes of the rigid body vector of system's states Euler angles angular velocity vector components of the angular velocity

\section{Introduction}

\subsection{Discovery of the phenomenon in space}

Vladimir Aleksandrovich Dzhanibekov is a famous Russian cosmonaut (shown in Fig. 1), who with his five space flights is recognized the champion in this category. In fact, he has spent in space 145 days, 15 hours and 35 seconds. ${ }^{2)}$ Time duration of his open space walks is 8 hours and 34 minutes.

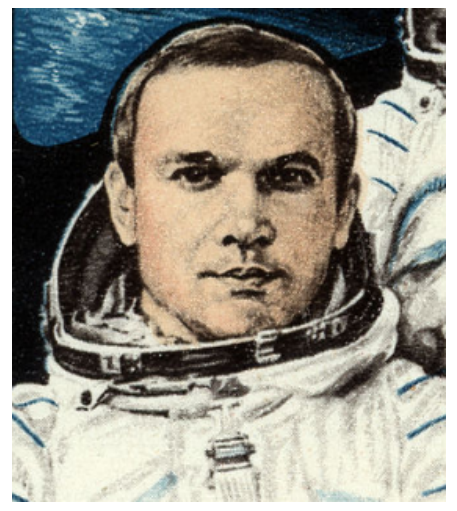

Fig. 1. Vladimir Aleksandrovich Dzhanibekov. ${ }^{1)}$

His first flight was in 1978. And during his fifth space flight, on 25-June-1985, he worked on-board of the space assembly "Salyut-7"-“Soyuz T-13", unpacking the payloads, delivered from the Earth by "Progress-24 (\#125)" supply transport vehicle. All arrived payloads, sent to the orbital space station "Salyut-7", were constrained to prevent their movements during the launch and flight of the space vehicle. The fixation 
of the payloads to the bases was secured by the classical fixation elements, involving long threaded rods and fixation "ear wing butterfly" nuts. The unpacking process involved exhausting unscrewing of many nuts, which required for each of the nuts to be rotated many times for them to travel significant distances along their corresponding fixing rods. To speed up the process, Vladimir Dzhanibekov has applied a significant torque impulse to the wing of the nut, which resulted in the initiation of the fast rotation of the nut and its conjugated translational motion along the threaded rod. The impulse was sufficient for the nut to complete unscrewing process on it own, and then to leave the rod. From this moment the nut continued its free flight, traveling along the axis of the left rod, while still being in rotation about this axis. After traveling the translational distance about $42 \mathrm{~cm}$, the nut, after its apparent stable and undisturbed flight, suddenly changed its axial orientation by 180 degrees, simultaneously changing its direction of rotating to opposite in the body-axis coordinate system and continued its flight backwards. It was even more amazing for the discoverer to realize, that this pattern of motion has been repeated in the periodic sequence, without any apparent external force applied. Using gymnastics terminology, it looked almost like the nut was performing the "Roundoff Backflip". This spectacular behaviour in the weightless environment of "flipping" of the rigid body on the axis of main rotation, later was named the "Dzhanibekov's effect" or "Dzhanibekov's phenomenon". Attracting attention of scientists and engineers, this discovery has even prompted a new hypothesis, that the Earth, similar to the "wing nut" in Dzhanibekov's phenomenon, is regularly performing its flips, but with a period of approximately 12,000 years. ${ }^{3)}$ In view of its potential importance, as a matter of precaution, the Dzhanibekov's discovery was classified by authorities for 10 years, ${ }^{4)}$ but after this term became public domain.

Surprisingly, the Dzhanibekov's phenomenon, which initially was perceived by some as counter-intuitive or even mysterious, has been conceptually predicted in 1971 by Beachley, ${ }^{5)}$ however has only been very recently explained in-depth in journal and on-line publications: ${ }^{6-9)}$ the Euler's equations have paved the theoretical ground to its scientific manifestation. Various popular videos/demos became available to wide audience. On numerous occasions, Vladimir A. Dzhanibekov himself explained his discovery in various lectures, TV programs and interviews. ${ }^{4,10)}$

The "Dzhanibekov's effect", and also so called "tennis racket phenomenon" were explained using Euler's equations for an unconstrained rigid body. ${ }^{7)}$ It has been realized that rotation of the body about the axis with intermediate principal moment of inertia becomes unstable, resulting in sudden change of its attitude.

\subsection{Demonstrations of the Dzhanibekov's phenomenon on board of the International Space Station (ISS)}

The Dzhanibekov's effect has been reproduced and observed during numerous demonstrations on board of ISS. Interested readers are referred to multiple videos in the media.

A series of experiments with various rigid bodies, including cylinders, cubes and right rectangular prisms was conducted on board of the ISS by Dan Burbank and Anton Shkaplerov, members of the 30-th expedition. ${ }^{11)}$

Astronaut Kevin Ford (NASA, 34-th expedition) and Japanese astronaut Koichi Wakata (JAXA, 38-th expedition), conducted experiments on board of ISS with spinning and tumbling pliers. ${ }^{12,13)}$

Richard Garriott, pioneer in commercial space travel, has also run a series of outreach program experiments on board of the ISS and in the video ${ }^{14)}$ demonstrates Dzhanibelkov's effect, using a deck of playing cards.

Another video on the topic shows a tumbling T-handle ${ }^{15)}$ experiment on board of the ISS and is a wonderful illustration of the instability of rotation about an asymmetric object's intermediate principal axis.

All these demonstrations could be explained with Euler's equation.

\subsection{Historical perspectives: Euler's equations}

Leonhard Euler (April 15, 1707 - Sept. 18, 1783) was a famous Swiss physicist and mathematician (the most eminent of the 18th century and one of the greatest in history), who made key contributions to various fields of mathematics and mechanics, leaving long-lasting heritage of more than 500 books and papers. It has been computed that his publications during his working life averaged about 800 pages a year. His portrait is presented in Fig. 2. Among numerous Euler's works, where

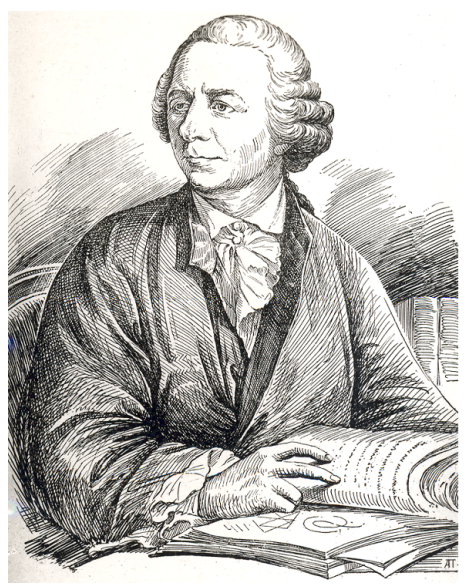

Fig. 2. L.Euler's portrait from the University of Tartu collection. ${ }^{16)}$

he developed rigid-body dynamics, very influential publication 17) has a very special place in history. It presented Euler's equations for the dynamics of a rigid body, widely used in modern engineering and science. In Fig. 3 we show the title of the publication, available from the Euler's archive ${ }^{18)}$ and the reproduced famous Euler's equations, exactly as they appeared in the original work. ${ }^{17)}$ In the equations in Fig. 3, $M$, accordingly to Euler, is the weight, and Euler emphasised that $M a a, M b b$ and $M c c$ are the inertia moments of the body along the three fixed axes and $P, Q$ and $R$ are the moments of the forces along the principal axes.

In modern language, the Euler's equations in Fig. 3(a) can be written as follows:

$$
\begin{aligned}
& \sum N_{x}=I_{x x} \dot{\omega}_{x}+\left(I_{z z}-I_{y y}\right) \omega_{y} \omega_{z} \\
& \sum N_{y}=I_{y y} \dot{\omega}_{y}+\left(I_{x x}-I_{z z}\right) \omega_{z} \omega_{x} \\
& \sum N_{z}=I_{z z} \dot{\omega}_{z}+\left(I_{y y}-I_{x x}\right) \omega_{x} \omega_{y}
\end{aligned}
$$

where $x, y, z$ are the principal axes of inertia fixed to the body; the components of angular velocity in this system are $\omega=\left(\omega_{x}\right.$, $\left.\omega_{y}, \omega_{z}\right)$, the torque is $N=\left(N_{x}, N_{y}, N_{z}\right)$ and the diagonal elements of the inertia tensor are $I_{x x}, I_{y y}$ and $I_{z z}$. 


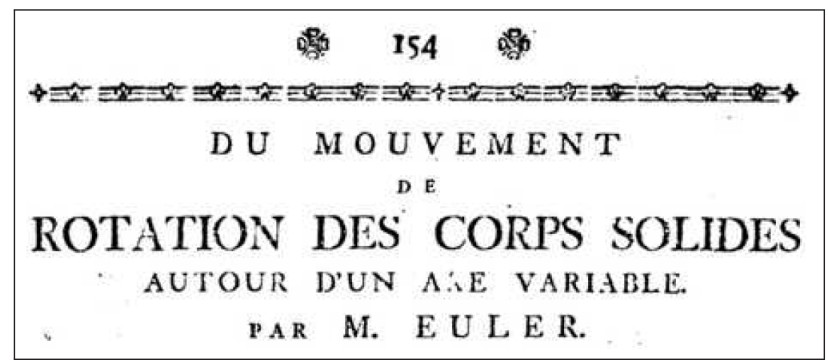

(a) The title of the historic L.Euler's work 17), dated by 1758 .

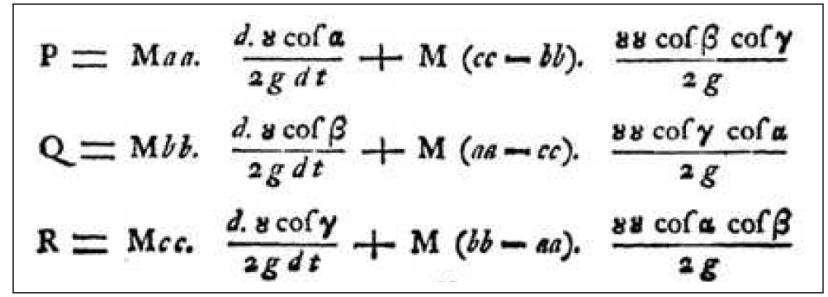

(b) Euler's equations as they appeared in the original L.Euler's work 17)

Fig. 3. Famous Euler's equations for the rigid body dynamics. ${ }^{18)}$

The equations, known as "Euler's equations", referred to as principal inertia axes, and with the angular velocity components in terms of the angles $\alpha, \beta, \gamma$, which are the angles subtended by the rotation axes with the principal ones fixed in the body.

\section{Numerical Simulation of the "Dzhanibekov's Effect"}

\subsection{Equations of motion}

Euler's equations (1), in the general case, can be applied for moments summed about any point $\mathcal{P}$, where $\mathcal{P}$ is a point on the rigid body that is attached to a fixed pivot in the inertial reference system. However, in this case the inertia properties should be calculated relative to the point $\mathcal{P}$.

In our study we will apply the Euler's equations for moments summed about the center of mass $\mathcal{G}$ of the rigid body, free from any external torques $\left(N_{x}=N_{y}=N_{z}=0\right)$ and in the further notations we will imply that $I_{x x}, I_{y y}, I_{z z}$ are principal moments of inertia of the body with respect to the $\mathcal{G}$ :

$$
\begin{aligned}
& I_{x x} \dot{\omega}_{x}-\left(I_{y y}-I_{z z}\right) \omega_{y} \omega_{z}=0 \\
& I_{y y} \dot{\omega}_{y}-\left(I_{z z}-I_{x x}\right) \omega_{z} \omega_{x}=0 \\
& I_{z z} \dot{\omega}_{z}-\left(I_{x x}-I_{y y}\right) \omega_{x} \omega_{y}=0
\end{aligned}
$$

The matrix form of the above Eq. (2) is:

$$
\left[\begin{array}{ccc}
I_{x x} & 0 & 0 \\
0 & I_{y y} & 0 \\
0 & 0 & I_{z z}
\end{array}\right]\left\{\begin{array}{c}
\dot{\omega}_{x} \\
\dot{\omega}_{y} \\
\dot{\omega}_{z}
\end{array}\right\}=\left\{\begin{array}{l}
\left(I_{y y}-I_{z z}\right) \omega_{y} \omega_{z} \\
\left(I_{z z}-I_{x x}\right) \omega_{z} \omega_{x} \\
\left(I_{x x}-I_{y y}\right) \omega_{x} \omega_{y}
\end{array}\right\}
$$

Let assume that the principal axes of the rigid body are coincident with the body fixed frame, then Euler angles $\psi, \theta$ and $\phi$ in the $z-y$ - $x$ form can be used to describe instantaneous orientation of a rigid body with respect to a fixed coordinate system :19)

$$
\begin{aligned}
& \omega_{x}=\dot{\psi} \sin \theta \sin \phi+\dot{\theta} \cos \phi \\
& \omega_{y}=\dot{\psi} \sin \theta \cos \phi-\dot{\theta} \sin \phi \\
& \omega_{z}=\dot{\psi} \cos \theta+\dot{\phi}
\end{aligned}
$$

which can also be written in the matrix form:

$$
\left[\begin{array}{ccc}
\sin \theta \sin \phi & \cos \phi & 0 \\
\sin \theta \cos \phi & -\sin \phi & 0 \\
\cos \theta & 0 & 1
\end{array}\right]\left\{\begin{array}{c}
\dot{\psi} \\
\dot{\theta} \\
\dot{\phi}
\end{array}\right\}=\left\{\begin{array}{c}
\omega_{x} \\
\omega_{y} \\
\omega_{z}
\end{array}\right\}
$$

For solving the rigid body dynamics problems, using numerical methods, we combine matrix equations (3) and (5) into a single equation:

$$
\begin{array}{r}
{\left[\begin{array}{cccccc}
I_{x x} & 0 & 0 & 0 & 0 & 0 \\
0 & I_{y y} & 0 & 0 & 0 & 0 \\
0 & 0 & I_{z z} & 0 & 0 & 0 \\
0 & 0 & 0 & \sin \theta \sin \phi & \cos \phi & 0 \\
0 & 0 & 0 & \sin \theta \cos \phi & -\sin \phi & 0 \\
0 & 0 & 0 & \cos \theta & 0 & 1
\end{array}\right]\left\{\begin{array}{c}
\dot{\omega}_{x} \\
\dot{\omega}_{y} \\
\dot{\omega}_{z} \\
\dot{\psi} \\
\dot{\theta} \\
\dot{\phi}
\end{array}\right\}=} \\
\\
\left\{\begin{array}{c}
\left(I_{y y}-I_{z z}\right) \omega_{y} \omega_{z} \\
\left(I_{z z}-I_{x x}\right) \omega_{z} \omega_{x} \\
\left(I_{x x}-I_{y y}\right) \omega_{x} \omega_{y} \\
\omega_{x} \\
\omega_{y} \\
\omega_{z}
\end{array}\right\}
\end{array}
$$

\subsection{Programming considerations}

Ordinary differential equations can be efficiently solved using Runge-Kutta methods. MATLAB ${ }^{\circledR}$ has a set of specialized procedures, including ode 45, ode23, ode113, ode15s, ode23s, ode $23 t$, ode $23 t b$, ode15i, to deal with various tasks, for example, described by the ordinary differential equation in the classical form: $\{\dot{\mathbf{x}}\}=\{f(t, \mathbf{x})\}$.

There is also a very useful option enabling solution of the problems, involving so called "mass" matrix $\mathcal{M}$ :

$$
[\mathcal{M}(t, \mathbf{x})]\{\dot{\mathbf{x}}\}=\{f(t, \mathbf{x})\}
$$

This option, accessible via the odeset, in some cases can improve efficiency and can also handle cases when the mass matrix is singular (non-invertible). As it can be seen, our Eqs. (6) correspond to the format given with Eq. (7), therefore, we use MATLAB ${ }^{\circledR}$ ode procedure in conjunction with the "mass matrix" option to simulate dynamic behaviour of the morphing spacecraft models.

\subsection{Study Case-1: results}

Let us consider a task of simulating the motion of the rigid body with the following parameters: $I_{x x}=0.3, I_{y y}=0.35$, $I_{z z}=0.4$ (all in $\left.\mathrm{kg}^{*} \mathrm{~m}^{2}\right)$, with the initial conditions ${ }_{i} \omega_{x}=0.1$, ${ }_{i} \omega_{y}=15,{ }_{i} \omega_{z}=0.1($ all in rad/s). Equations (6) were solved numerically and main results are given in Fig. 4. Their observation confirms periodical flipping of the system: indeed, $\omega_{y}$ in Fig. 4.(a) is periodically changing its sign.

It would be interesting to note that the period of the observed unstable motion can be estimated, using Eq. (37.12) in page 154 from the Ref. 20):

$$
T=4 K \sqrt{\frac{I_{x x} I_{y y} I_{z z}}{\left(I_{z z}-I_{y y}\right)\left(H^{2}-2 \mathcal{K}_{0} I_{x x}\right)}}
$$

where $K$ is complete elliptic integral of the first kind

$$
K=\int_{0}^{1} \frac{d s}{\sqrt{\left(1-s^{2}\right)\left(1-k^{2} s^{2}\right)}}=\int_{0}^{\pi / 2} \frac{d u}{\sqrt{1-k^{2} \sin ^{2} u}}
$$

being a function of the parameter $k^{2}$

$$
k^{2}=\frac{\left(I_{y y}-I_{x x}\right)\left(2 \mathcal{K}_{0} I_{z z}-H^{2}\right)}{\left(I_{z z}-I_{y y}\right)\left(H^{2}-2 \mathcal{K}_{0} I_{x x}\right)}
$$




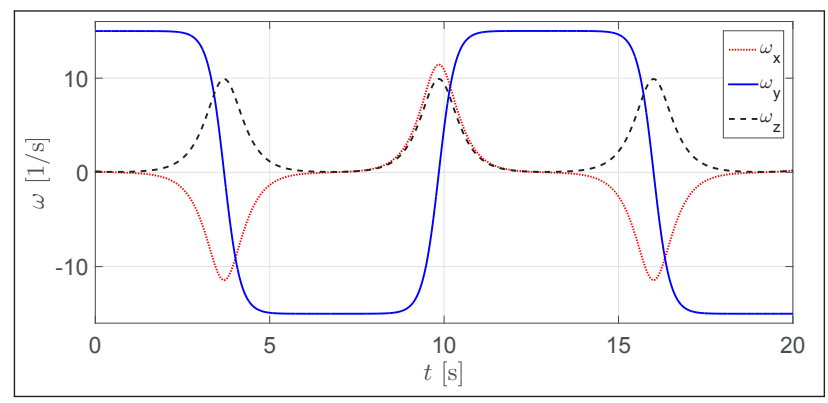

(a) Time histories of the angular velocity components of the spacecraft.

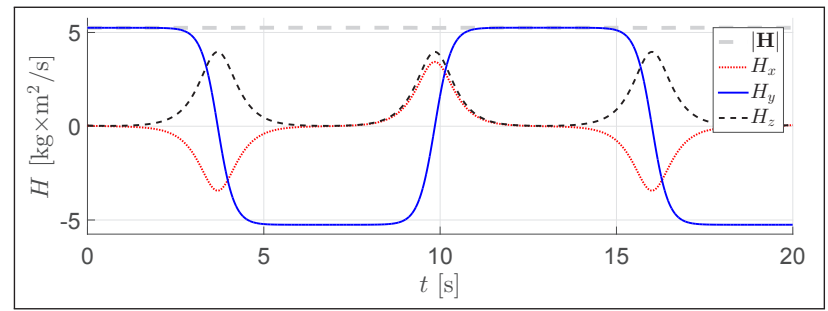

(b) Time histories of the angular momenta of the spacecraft.

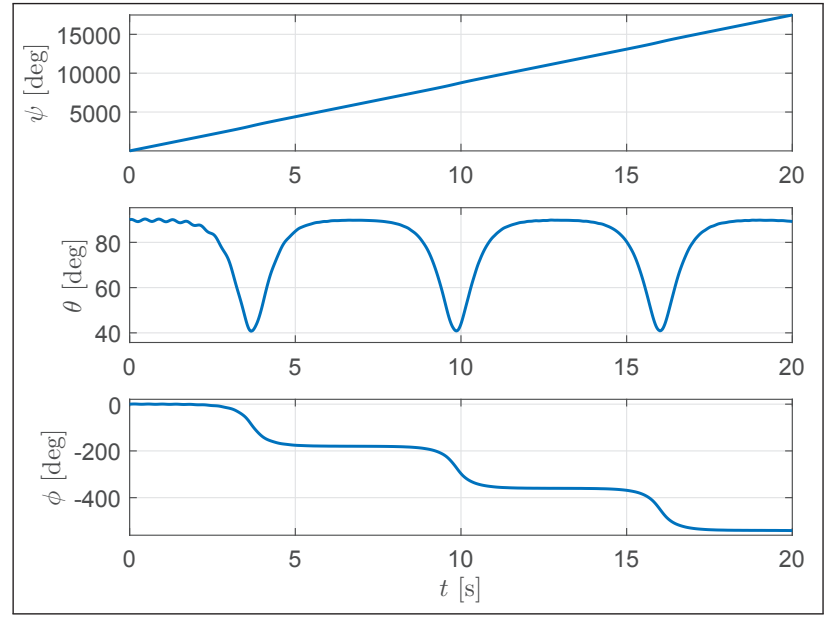

(c) Time histories of the Euler angles.

Fig. 4. Dzhanibekov's effect: simulation results for the study Case-1.

It should be noted that Eqs. (8)-(10) correspond to the case of $H^{2}>2 \mathcal{K}_{0} I_{y y}$. If this condition is not observed, in Eqs. (8)-(10) subscripts " $x x$ " and " $z z$ " must be interchanged.

As calculation of $K$ is very sensitive to the input data, high precision calculation methods and tools would be advisable. If the accuracy of MATLAB ${ }^{\circledR}$ and Wolfram MATHEMATICA ${ }^{\circledR}$ is deemed insufficient, using a specialized precision elliptic integral calculations, ${ }^{21)}$ can provide accuracy of up to 50 digits.

Using the study Case-1 data and Eqs. (8)-(10), and result for $K$ with 22-digit accuracy $(K=6.66504144391522819056)$ enables us to calculate $T=12.33 \mathrm{~s}$, which is identical to the observed in numerical simulation, presented in Fig. 4(b).

Figure 4(b) confirms that during the "flipping" motion, the angular momentum in the system is conserved. At last, Fig. 4(c) shows that while $\psi$ is monotonically increasing, the $\phi$ pattern is quite different: there are evident "plateau" segments corresponding to small changes in $\phi$ around $0^{\circ}, 180^{\circ}, 360^{\circ}$, etc. However, the most important observation in the context of this paper is presence of the multiple zero-crossings for various components of the angular velocity, in particular, for $\omega_{x}$ and $\omega_{y}$ in the test case.

\section{Proposing New Spacecraft Designs/Missions, Utilising Dzhanibelov's Phenomenon}

\subsection{Proposing Idea of "Switching ON/OFF" Dzhanibekov's spacecraft flipping motion by con- trolled morphing of the tumbling object}

Flipping motion of the rigid body, during which the direction of the angular velocity of the main rotation, let say, $\omega_{y}$, is intermittently changing to opposite, is called "Dzhanibekov's effect". It is a consequence of the moment of inertia, associated with the main rotation, being between two other values of the moments of inertia, $I_{x x}$ and $I_{z z}$, in other words, having an intermediate value among principal moments of inertia.

What if there is a need to stop or suspend for some time the unstable "flipping" motion of the object? For this purpose, Beachley, ${ }^{5)}$ propsed four types of mass translations. However, this proposition was rather conceptual, as did not involve equations of motion of the spacecraft with variable interial properties, did not explain the change in moments of inertia, and did not investigate the inversion timing. We have addressed all these issues in a systematic manner and for the purpose of control of the spacecraft attitude, propsed general method of inertial morphing of the spacecraft, mathematically linking these modifications to the changes in moments of inertia and simulating transitional spacecraft response, using rigorous equations of motion. ${ }^{22)}$ In particular, we showed, that for stabilization of the spacecraft, there exist two morphing strategies, after implementation of which the intermediate moment of inertia becomes the smallest or largest among all principal moments of inertia.

In the illustration case, where we selected $y$ axis to be the axis of the main rotation, the condition for the unstable "Dzhanibekov's effect"-type motion can be written as:

$$
I_{x x}<I_{y y}<I_{z z}
$$

However, if via special design of the spacecraft, enabling the change of its principal moments of inertia (via mechanical or other means), the targeted value of $I_{y y}$ is in controllable way forcefully "moved" outside the embrace of $I_{x x}$ and $I_{z z}$, then the condition of instability Eq. (11) would no longer be satisfied and the unstable motion would be "switched OFF"! Conceptually, this proposition can be illustrated with the dia-

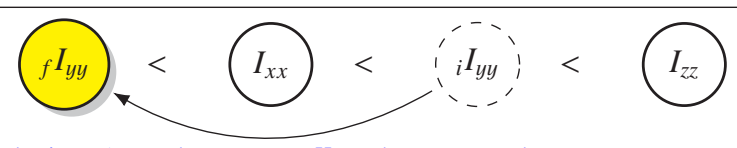

Solution-1: make $I_{y y}$ smaller than $I_{x x}$ and $I_{z z}$

Solution-2: make $I_{y y}$ larger than $I_{x x}$ and $I_{z z}$

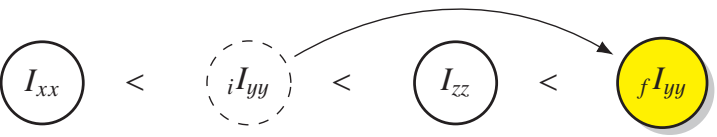

Fig. 5. Possible conceptual solutions for stabilizing an unstable spacecraft with its main rotation about the $y$ axis.

gram in Fig. 5, which presents two solutions. The first conceptual solution involves reduction of initial value of $I_{y y}$ (which we denote as ${ }_{i} I_{y y}$ ) to its new (or final) value ${ }_{f} I_{y y}$, being smaller than 
$I_{x x}$ value. And the second solution involves increase of the initial value of $I_{y y}$ (which we denote as ${ }_{i} I_{y y}$ ) to its new value ${ }_{f} I_{y y}$, being larger than $I_{z z}$.

For the numerical verification of the concept, let us assume the following demonstration values: $I_{x x}=0.3, I_{y y}=0.35$ and $I_{z z}=0.4$ (all in $\mathrm{kg}^{*} \mathrm{~m}^{2}$ ), which are conforming with the general condition Eq. (11) of the flipping unstable motion, which would result if the main rotation about $y$ axis is initiated. And in this case, in order to test the concept of "switching OFF" the flipping motion, we will change ${ }_{i} I_{y y}=0.35$ to its new value of ${ }_{f} I_{y y}=0.2$ (solution-1) or ${ }_{f} I_{y y}=0.5$ (solution-2).

However, in order to proceed with the numerical simulations, we need to expand the Euler equations, allowing variations in the moments of inertia of the rigid body.

\subsection{Equations of motion: extending Euler's equations}

In order to simulate the cases of the morphing spacecraft with variable moments of inertia, we need to extend classic Euler's Eqs. (1). We note that the sum of the moments about the center of mass of a rigid body due to external forces and couples equals to the rate of change of the angular momentum about the center of mass : ${ }^{19)}$

$$
\sum \overrightarrow{\mathbf{N}}=\left.\frac{d \overrightarrow{\mathbf{H}}}{d t}\right|_{\text {Inertial }}=\left.\frac{d \overrightarrow{\mathbf{H}}}{d t}\right|_{\text {Body }}+\vec{\omega} \times \overrightarrow{\mathbf{H}}
$$

Also, the components of the angular momentum vector, $\overrightarrow{\mathbf{H}}(t)$, with respect to the body-axis frame can be expressed by the product between the principal moment of inertia matrix $I_{\mathcal{G}}$ and the components of the angular velocity vectors as follows:

$$
\overrightarrow{\mathbf{H}}(t)=\left[\begin{array}{ccc}
I_{x x} & 0 & 0 \\
0 & I_{y y} & 0 \\
0 & 0 & I_{z z}
\end{array}\right]\left\{\begin{array}{l}
\omega_{x} \\
\omega_{y} \\
\omega_{z}
\end{array}\right\}
$$

Therefore, extended Euler's equations can now be written as:

$$
\begin{aligned}
& {\left[\begin{array}{ccc}
\dot{I}_{x x} & 0 & 0 \\
0 & \dot{I}_{y y} & 0 \\
0 & 0 & \dot{I}_{z z}
\end{array}\right]\left\{\begin{array}{c}
\omega_{x} \\
\omega_{y} \\
\omega_{z}
\end{array}\right\}+\left[\begin{array}{ccc}
I_{x x} & 0 & 0 \\
0 & I_{y y} & 0 \\
0 & 0 & I_{z z}
\end{array}\right]\left\{\begin{array}{l}
\dot{\omega}_{x} \\
\dot{\omega}_{y} \\
\dot{\omega}_{z}
\end{array}\right\}+(12)} \\
& {\left[\begin{array}{ccc}
0 & -\omega_{z} & \omega_{y} \\
\omega_{z} & 0 & -\omega_{x} \\
-\omega_{y} & \omega_{x} & 0
\end{array}\right]\left[\begin{array}{ccc}
I_{x x} & 0 & 0 \\
0 & I_{y y} & 0 \\
0 & 0 & I_{z z}
\end{array}\right]\left\{\begin{array}{l}
\omega_{x} \\
\omega_{y} \\
\omega_{z}
\end{array}\right\}=\left\{\begin{array}{l}
0 \\
0 \\
0
\end{array}\right\}}
\end{aligned}
$$

For solving the morphing rigid body dynamics problems, using numerical methods, we combine matrix Eqs. (12) and (5) into a single equation:

$$
\begin{gathered}
{\left[\begin{array}{cccccc}
I_{x x} & 0 & 0 & 0 & 0 & 0 \\
0 & I_{y y} & 0 & 0 & 0 & 0 \\
0 & 0 & I_{z z} & 0 & 0 & 0 \\
0 & 0 & 0 & \sin \theta \sin \phi & \cos \phi & 0 \\
0 & 0 & 0 & \sin \theta \cos \phi & -\sin \phi & 0 \\
0 & 0 & 0 & \cos \theta & 0 & 1
\end{array}\right]\left\{\begin{array}{c}
\dot{\omega}_{x} \\
\dot{\omega}_{y} \\
\dot{\omega}_{z} \\
\dot{\psi} \\
\dot{\theta} \\
\dot{\phi}
\end{array}\right\}=} \\
\left\{\begin{array}{c}
\left(I_{y y}-I_{z z}\right) \omega_{y} \omega_{z}-\dot{I}_{x x} \omega_{x} \\
\left(I_{z z}-I_{x x}\right) \omega_{z} \omega_{x}-\dot{I}_{y y} \omega_{y} \\
\left(I_{x x}-I_{y y}\right) \omega_{x} \omega_{y}-\dot{I}_{z z} \omega_{z} \\
\omega_{x} \\
\omega_{y} \\
\omega_{z}
\end{array}\right\}
\end{gathered}
$$

Equations (13) are the main equations, used in this paper and solved using ode MATLAB ${ }^{\circledR}$ Runge-Kutta solver, with "mass matrix" option, as per Eq. (7).

\section{Proposing "Geometric Morphing" of Spacecraft: Con- ceptual Considerations}

To demonstrate the feasibility of the controllable behaviour of the spacecraft, let us consider a simple conceptual model of the morphing spacecraft, constructed as an axisymmetric set of three orthogonal dumbbells, each of which has negligible mass of the rod, connecting two equal concentrated masses at its ends. Let us also assume, for conceptual simplicity, that three dumbbells are connected at the middle points of their rods, and the corresponding masses $m_{x}, m_{y}$ and $m_{z}$ are located at the distances $r_{x}, r_{y}$ and $r_{z}$ from the axes of rotation $x, y$ and $z$, as shown in Fig. (6). In the illustrated conceptual design, morphing of the spacecraft is achieved via independent synchronized control of the position coordinates $r_{x}=r_{x}(t), r_{y}=r_{y}(t)$ and $r_{z}=r_{z}(t)$ of the masses $m_{x}, m_{y}$ and $m_{z}$.

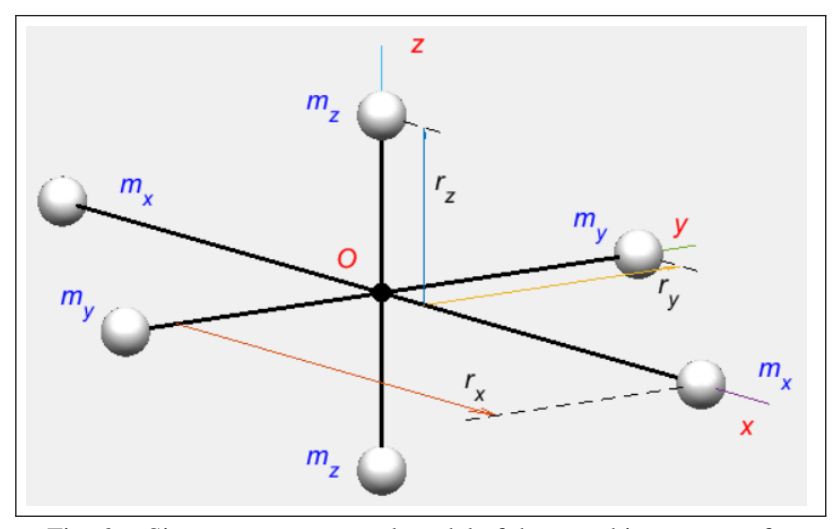

Fig. 6. Six-masses conceptual model of the morphing spacecraft.

The principal moments of inertia of the system can be calculated as follows:

$$
\begin{aligned}
& I_{x x}=2 m_{y} r_{y}^{2}+2 m_{z} r_{z}^{2} \\
& I_{y y}=2 m_{z} r_{z}^{2}+2 m_{x} r_{x}^{2} \\
& I_{z z}=2 m_{x} r_{x}^{2}+2 m_{y} r_{y}^{2}
\end{aligned}
$$

Then by adding all equations in (14), we can get:

$$
\frac{1}{2}\left(I_{x x}+I_{y y}+I_{z z}\right)=2\left(m_{x} r_{x}^{2}+m_{y} r_{y}^{2}+m_{z} r_{z}^{2}\right)
$$

Then, subtracting from Eq. (15) consecutively each of Eq. (14), we can get:

$$
\begin{aligned}
& r_{x}=\sqrt{\left(I_{y y}+I_{z z}-I_{x x}\right) /\left(4 m_{x}\right)} \\
& r_{y}=\sqrt{\left(I_{z z}+I_{x x}-I_{y y}\right) /\left(4 m_{y}\right)} \\
& r_{z}=\sqrt{\left(I_{x x}+I_{y y}-I_{z z}\right) /\left(4 m_{z}\right)}
\end{aligned}
$$

Let us assume, for the illustration purpose, that $m_{x}=m_{y}=$ $m_{z}=1 \mathrm{~kg}, I_{x x}=0.3 \mathrm{~kg}^{*} \mathrm{~m}^{2}, I_{x x}=0.35 \mathrm{~kg}^{*} \mathrm{~m}^{2}, I_{x x}=0.4$ $\mathrm{kg}^{*} \mathrm{~m}^{2}$. Then, for the case of the tumbling spacecraft considered in Section 2.3, we can find the initial radial positions of the spacecraft masses, using Eqs. (16):

$$
{ }_{i} r_{x}=0.2500 \mathrm{~m} ;{ }_{i} r_{y}=0.2958 \mathrm{~m} ;{ }_{i} r_{z}=0.3354 \mathrm{~m} .
$$

These values for the unit masses would ensure, that the inertial properties of the spacecraft are $I_{x x}=0.3 \mathrm{~kg}^{*} \mathrm{~m}^{2}, I_{y y}=0.35$ $\mathrm{kg} * \mathrm{~m}^{2}, I_{z z}=0.4 \mathrm{~kg} * \mathrm{~m}^{2}$. Note that in our example here $I_{y y}$ 


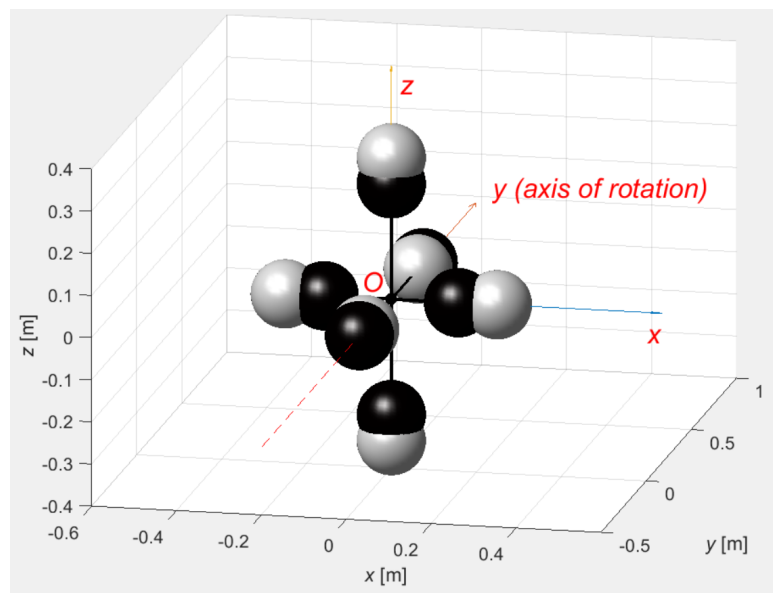

(a) Solution-1, as per Fig. 5 .

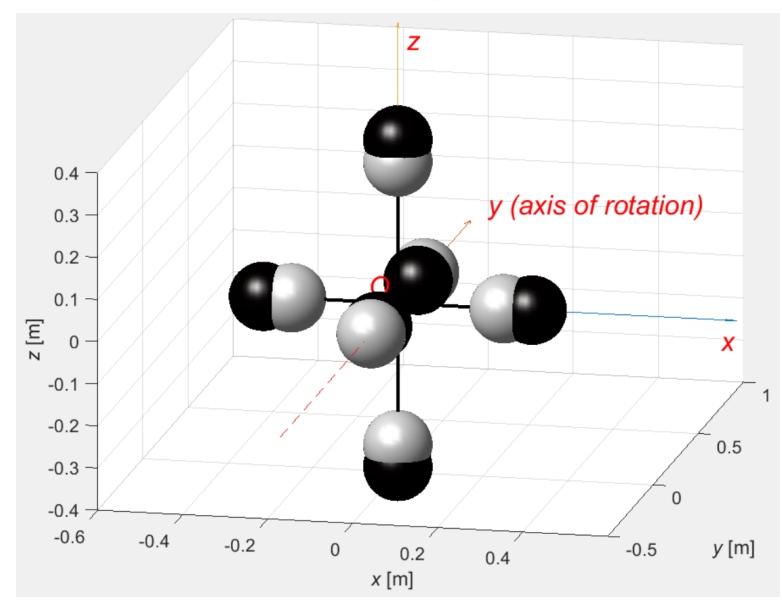

(b) Solution-2, as per Fig. 5.

Fig. 7. Conceptual 6-masses design of the morphing spacecraft, capable of self-transfer from unstable "Dzhanibekov's effect"-type flipping motion to stable motion (and vice versa): white spheres - unstable configuration for $y$ main rotation, black spheres - stable configuration.

has an intermediate value among all principal moments of inertia: $I_{x x}<I_{y y}<I_{z z}$, therefore if the spacecraft is provided with the initial angular velocities $\omega_{x}=\omega_{z}=0.1 \mathrm{rad} / \mathrm{s}$ and $\omega_{y}=15 \mathrm{rad} / \mathrm{s}$, with the prevailing rotation about $y$ body axis, then the spacecraft rotation about this axis would be unstable and classical "Dzhanibekov's effect" periodic flipping would be observed.

It will be shown in Section 5.1, that if during the "flipping" motion, at the instant, when the angular velocities $\omega_{x}=\omega_{z}$ are close to zeros, the moment of inertia ${ }_{i} I_{y y}=0.35 \mathrm{~kg}^{*} \mathrm{~m}^{2}$ is rapidly changed to its new value of ${ }_{f} I_{y y}=0.2 \mathrm{~kg}^{*} \mathrm{~m}^{2}$, then the nature of the followed motion of the system would change from unstable "flipping" to stable. We call it "switching OFF" the flipping motion.

This will be occurring because the moment of inertia $I_{y y}$ stops being the intermediate value and the rotation about $y$ body axis is becoming stable, without changes in the direction of $\omega_{y}$.

The new values of the position radii, corresponding to the "solution-1" in Fig. 5, can be calculated using Eqs. (16):

$$
{ }_{f} r_{x}=0.1581 \mathrm{~m} ;{ }_{f} r_{y}=0.3536 \mathrm{~m} ;{ }_{f} r_{z}=0.2739 \mathrm{~m}
$$

The spacecraft masses at these radius positions are shown in Fig. 7(a) with dark color.

The flipping motion can be also stopped, using "solution-2", shown in conceptual Fig. 5. For the purpose of the illustration
Table 1. Summary of two solution for the spacecraft inertial morphing, stopping flipping motion of the spacecraft, initially being in the "Dzhanibekov's effect" "flipping" mode.

\begin{tabular}{|c|c|c|c|c|c|c|}
\hline \multicolumn{7}{|c|}{ Solution-1: } \\
\hline & $r_{x}$ & $r_{y}$ & $r_{z}$ & $I_{x x}$ & $I_{y y}$ & $I_{z z}$ \\
\hline Initial & 0.2500 & 0.2958 & 0.3354 & 0.30 & 0.35 & 0.40 \\
\hline Final & 0.1581 & 0.3536 & 0.2739 & 0.30 & 0.20 & 0.40 \\
\hline \multicolumn{7}{|c|}{ Solution-2: } \\
\hline & $r_{x}$ & $r_{y}$ & $r_{z}$ & $I_{x x}$ & $I_{y y}$ & $I_{z z}$ \\
\hline Initial & 0.2500 & 0.2958 & 0.3354 & 0.30 & 0.35 & 0.40 \\
\hline Final & 0.3162 & 0.2236 & 0.3873 & 0.30 & 0.50 & 0.40 \\
\hline
\end{tabular}

of the concept, let us consider rapid increase of the $I_{y y}$ from its initial value of ${ }_{i} I_{y y}=0.35 \mathrm{~kg} * \mathrm{~m}^{2}$ to its new value of ${ }_{f} I_{y y}=0.5$ $\mathrm{kg} * \mathrm{~m}^{2}$. The new values of the position radii, corresponding to the "solution-2" in Fig. 5, can be calculated using Eqs. (16):

$$
{ }_{f} r_{x}=0.3162 \mathrm{~m} ;{ }_{f} r_{y}=0.2236 \mathrm{~m} ;{ }_{f} r_{z}=0.3873 \mathrm{~m} \text {. (19) }
$$

The spacecraft masses at these radius positions are shown in Fig. 7(b) with dark color.

The morphing of the spacecraft from the initially unstable configuration [as per Eq. (17)], associated with the "flipping" motion, to its final stable configuration [as per Eq. (18) or (19) and Solution-1 or 2 in Fig. 5], are shown in Fig. 7, where masses for the initial configuration are shown in white, whereas the masses for the final configuration are shown in black color.

Summary for both solutions is presented in Table 1. It would be important to note, that in the presented cases, it was not obligatory during the morphing of the system and its transition from the "initial" to "final" states to keep both values of $I_{x x}$ and $I_{z z}$ unchanged. However, it was done for purpose to emphasize the role of the $I_{y y}$ in the process of stabilization of the system.

\section{Motion of Spacecraft with Geometric Morphing}

\subsection{Study Case-2: "Switching OFF" flipping motion of the spacecraft after one flip (solution-1)}

Figure 4 shows that at the instant $t=6.77 \mathrm{~s}$, the angular velocity $\omega_{y}$ has its highest value and $\omega_{x}$ changes its value from negative to positive. It is believed that this instant, corresponding to the most prominent rotation about $y$-body axis, would be the best time to apply morphing to the spacecraft. In our demo case the moment of inertia $I_{y y}$ is changed from 0.35 to 0.2 , as per Fig. 8 within relatively short period of time of $0.2 \mathrm{~s}$. Results of the simulation are given with Fig. 10. Figure 10(a) shows that the simulated morphing led to the step-type increase of the angular velocity $\omega_{2}$ of the body and did not initiate significant oscillations in $\omega_{x}$ and $\omega_{z}$. In contrast to Case-1, where $\omega$ and $H$ plots had similar shapes, in the Case- 2 these plots are different. Figure 10(b) shows that morphing did not change the angular momentum $H_{y}$ and after the morphing was completed, the value of $H_{y}$ stayed almost unchanged, evidencing that attempt to stop the "flipping" motion was successful. At last, note that as the stabilised value of $\phi=180^{\circ}$, the stabilized spacecraft is flying backwards, with its initial heading attitude changed by $180^{\circ}$ !

\subsection{Study Case-3: "Switching OFF" flipping motion of} the spacecraft after one flip (solution-2)

It is interesting to observe that stabilization of the system, illustrated with Figs. 10 has been achieved with a controllable 


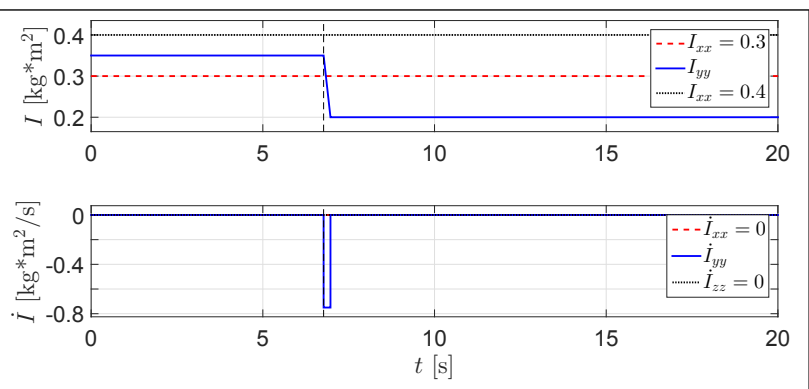

Fig. 8. Time history of the controlled manipulation with the moment of inertia $I_{y y}$ to stop flipping motion of the system (Case-2).

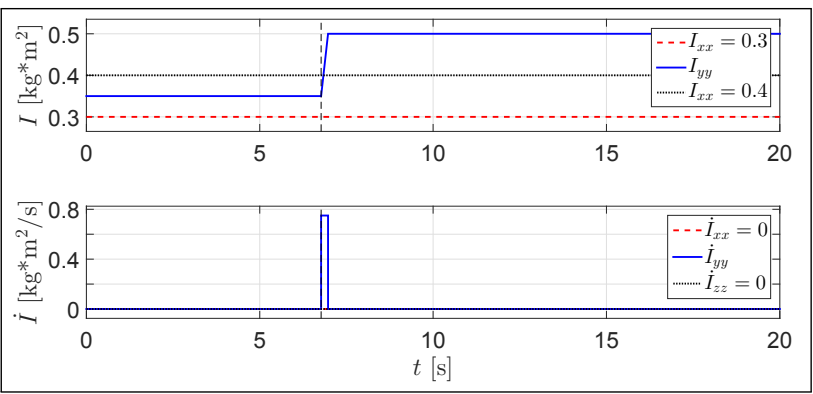

Fig. 9. Time history of the controlled manipulation with the moment of inertia $I_{y y}$ to stop flipping motion of the system (Case-3).

change of the moment of inertia $I_{y y}$ (associated with the main rotation of the spacecraft), which initially had its value of ${ }_{i} I_{y y}=$ 0.35 , being an intermediate value, surrounded by the smallest $I_{x x}=0.2$ and largest $I_{z z}=0.4$ moments of inertia:

$$
I_{x x}<{ }_{i} I_{y y}<I_{z z}
$$

While keeping values of $I_{x x}$ and $I_{z z}$ unchanged, the value of $I_{y y}$ in the presented experiment was changed from ${ }_{i} I_{y y}=0.35$ to the final value of ${ }_{f} I_{y y}=0.5$, as per Fig. 8, after which it became the largest principal moment of inertia:

$$
I_{x x}<I_{z z}<{ }_{f} I_{y y}
$$

Figure 10 shows that as one of the consequences of the increase of $I_{y y}$, was a reduction from 15 to $10.5 \mathrm{rad} / \mathrm{s}$ of the associated angular velocity $\omega_{y}$ of the spacecraft. This simulation result is in perfect agreement with the conservation of the angular momentum of the system, suggesting that $\omega_{y}$ must be reduced by the ratio of $15 \times\left({ }_{i} I_{y y} / f I_{y y}\right)=15 \times(0.35 / 0.5)=10.5 \mathrm{rad} / \mathrm{s}$.

In contrast to Case- 1 , where $\omega$ and $H$ plots had similar shapes, in the Case-3 these plots are different. Figure 11(b) shows that morphing did not change the angular momentum $H_{y}$ and after the morphing was completed, the value of $H_{y}$ stayed almost unchanged, evidencing that the stopping "flipping" motion has been successful.

\subsection{Study Case-4: "Switching OFF" flipping motion of the spacecraft after two flips (solution-1)}

We now demonstrate switching OFF the "flipping" motion of the morphing spacecraft after it performs two flips. The time history of morphing is similar to presented in Fig. 8, but morphing is starting at $t=13.54 \mathrm{~s}$. Results of this Case- 4 are presented in Fig. 12. Observed reduction of the angular velocity $\omega_{y}$ is the same, as for the Case-2, however, in Case-2 after the motion is stabilized, the spacecraft continues its flight backwards, whereas in the current case, the stabilized attitude of the spacecraft is the same as at the initial time.

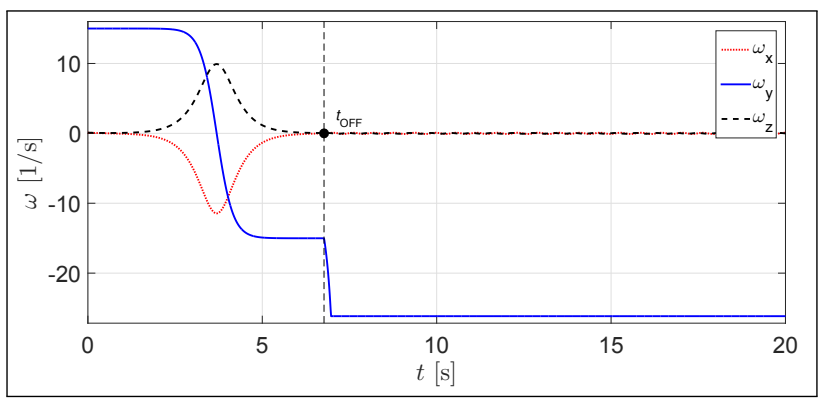

(a) Time histories of the angular velocity components of the spacecraft.

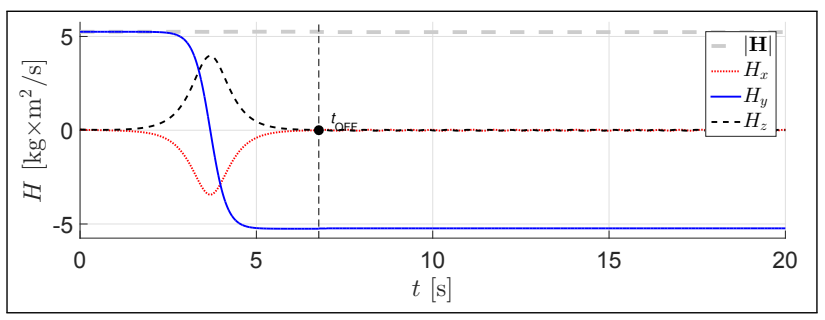

(b) Time histories of the angular momenta of the spacecraft.

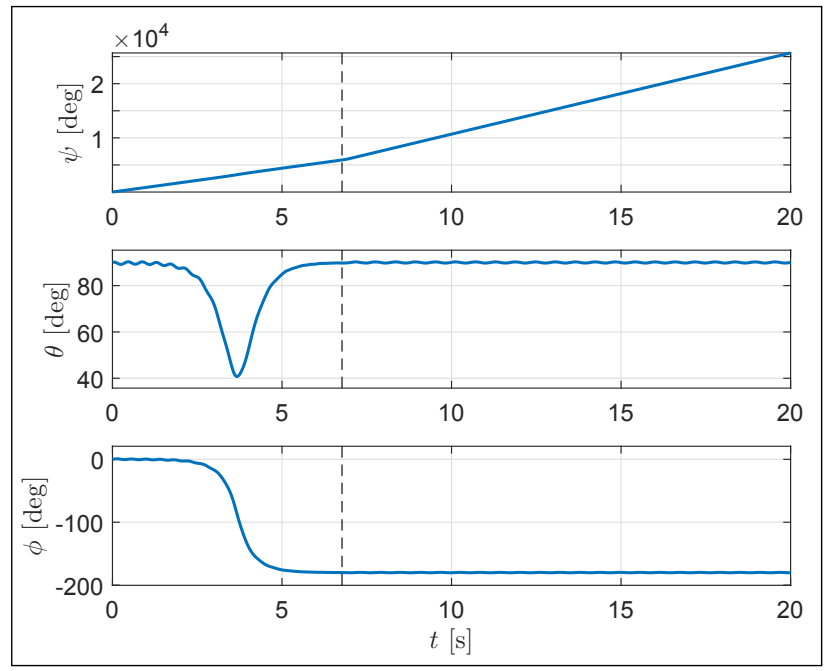

(c) Time histories of the Euler angles.

Fig. 10. Simulation results for Case-2: switching off the "flipping" motion of the spacecraft. Note: $t_{O F F}$ is an instant, at which the "flipping" motion of the system was stopped, or, in other words, "switched OFF".

\subsection{Study Case-5: "Switching ON" spacecraft flipping motion}

In a similar way as stabilization, described in the Cases 24 was achieved, we can initiate the "flipping" motion of the spacecraft. For this, the axis of the major rotation of the system (let say, $y$ ) initially should coincide with the axis of minimal or maximal moments of inertia, i.e. one of the conditions should be satisfied: $I_{y y}<\min \left(I_{x x}, I_{z z}\right)$ or $I_{y y}>\max \left(I_{x x}, I_{z z}\right)$. In this case initiated rotation would be stable, without "flipping". To activate the "flipping" motion, morphing of the system should be performed, which should result in $I_{y y}$ becoming an intermediate value between $I_{x x}$ and $I_{z z}$. In the study Case-5, as illustration, we use the following values: $\omega_{x}=0.1, \omega_{y}=26.25, \omega_{z}=0.1$ (all - in rad/s), $I_{x x}=0.3,{ }_{i} I_{y y}=0.2,{ }_{f} I_{y y}=0.35, I_{z z}=0.4$ (all - in $\mathrm{kg}^{*} \mathrm{~m}^{2}$ ). The time history of applied morphing is presented in Fig. 13 and the results of the simulation are shown in Fig. 14. 


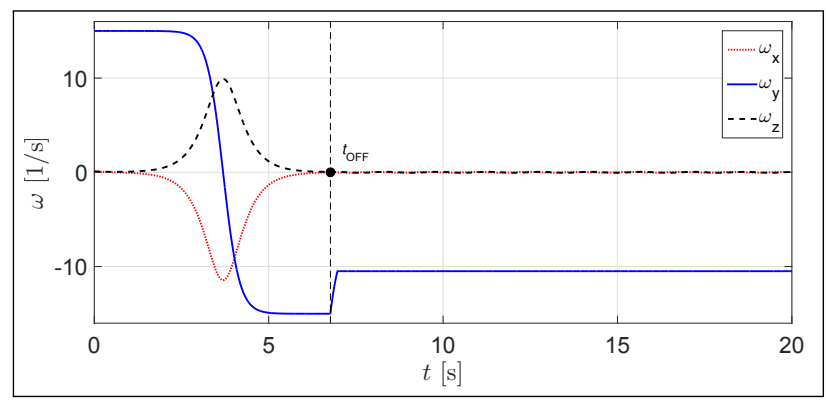

(a) Time histories of the angular velocity components of the spacecraft.

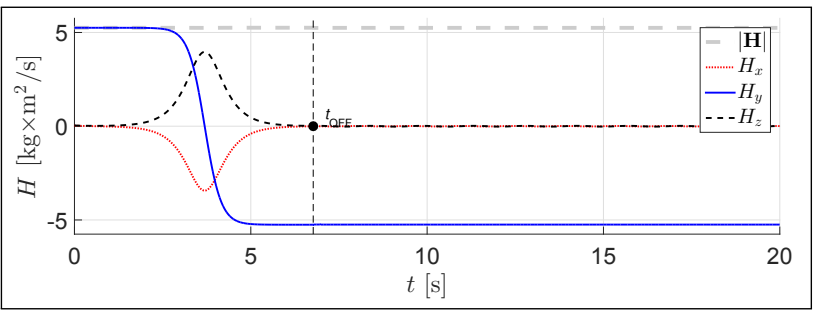

(b) Time histories of the angular momenta of the spacecraft.

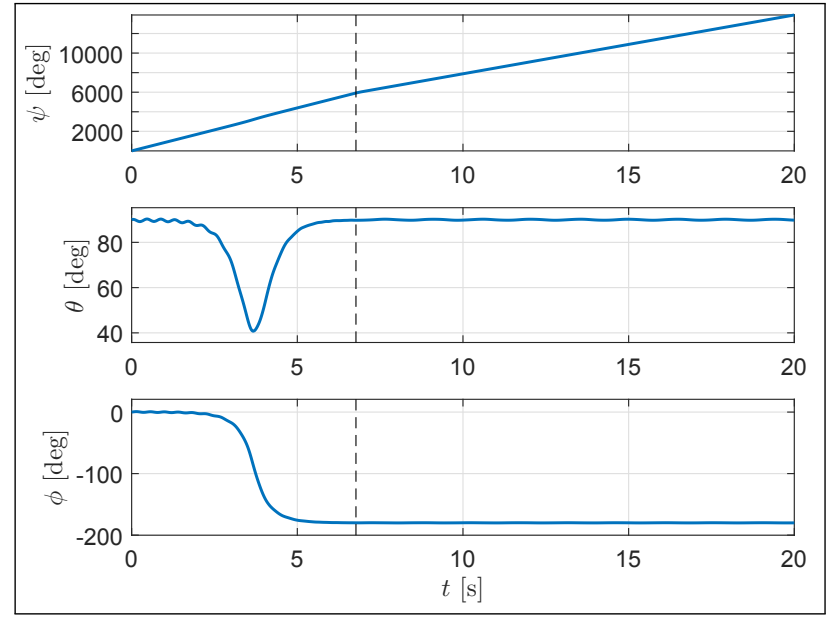

(c) Time histories of the Euler angles.

Fig. 11. Simulation results for the study Case-3: switching off the "flipping" motion of the spacecraft.

\subsection{Study Case-6: "Switching ON" spacecraft flipping motion with following one flip and "Switching OFF"}

Case- 6 represents further development of the Case- 5 by switching OFF the "flipping" motion at $t=9.89 \mathrm{~s}$, instant of the maximal value of $\omega_{y}$. The time history of applied morphing is presented in Fig. 15 and the results of the simulation are shown in Fig. 16.

\subsection{Study Case-7: Control of the frequency of the flipping motion via "inertial morphing"}

Case-7 demonstrates ability of the proposed "inertia morphing" for control of the frequency of the "flipping" motion. In this demo, inertia properties of the "flipping" system ${ }_{i} I_{x x}=0.3$, ${ }_{i} I_{y y}=0.395,{ }_{i} I_{z z}=0.4$, were changed after three $180^{\circ}$ flips, to ${ }_{f} I_{x x}=0.3,{ }_{f} I_{y y}=0.35,{ }_{f} I_{z z}=0.4$ (all in $\mathrm{kg}^{*} \mathrm{~m}^{2}$ ), as per Fig. 17. However, the intermediate value of the moment of inertia $I_{y y}$ was still kept within the values of $I_{x x}$ and $I_{z z}: I_{x x}<I_{y y}<I_{z z}$. It is really interesting to observe that the it was possible to achieve substantial change of the frequency of the flipping motion. Calculations of the flipping motion periods before and after "morphing" can be calculated, using Eq. (8): $T_{i}=21.5 \mathrm{~s}$ and $T_{f}=10.8$ $\mathrm{s}$ (twice reduction!). This is in perfect correspondence with the

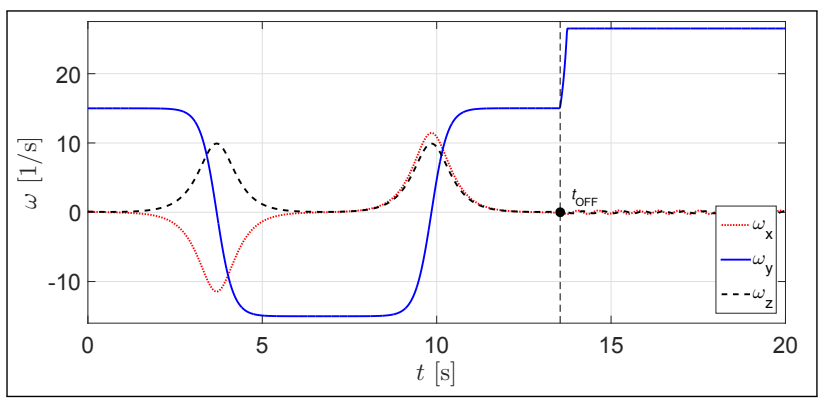

(a) Time histories of the angular velocity components of the spacecraft.

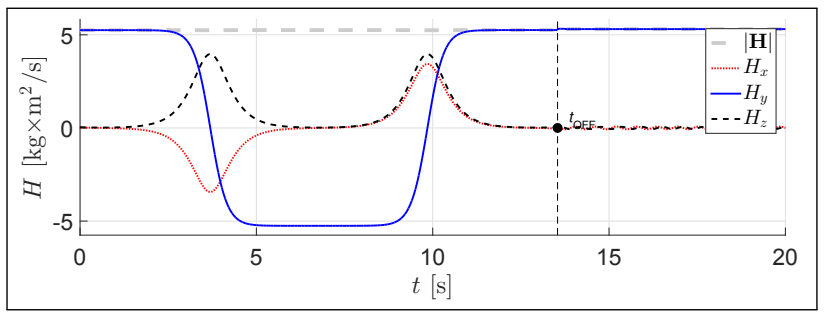

(b) Time histories of the angular momenta of the spacecraft.

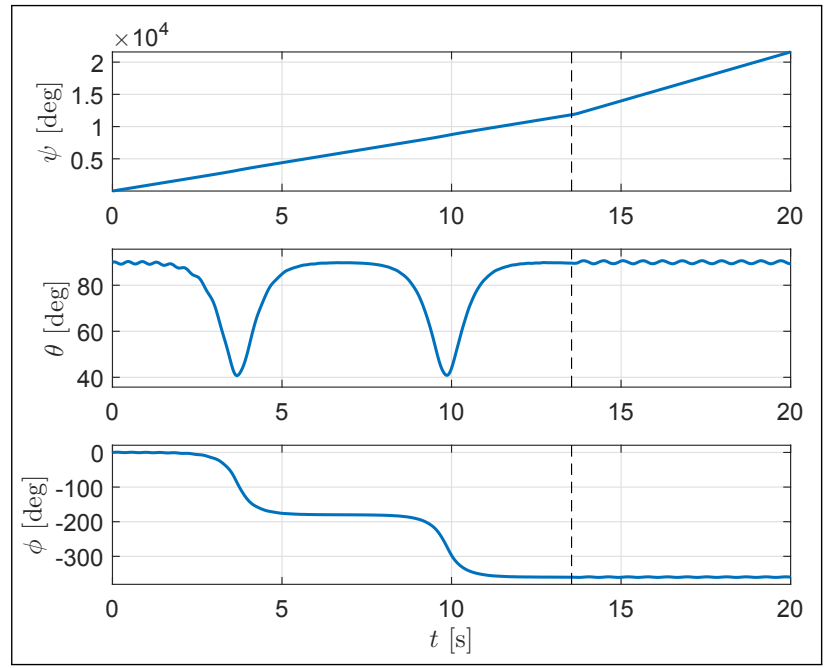

(c) Time histories of the Euler angles.

Fig. 12. Simulation results for the study Case-4: switching off the "flipping" motion of the spacecraft.

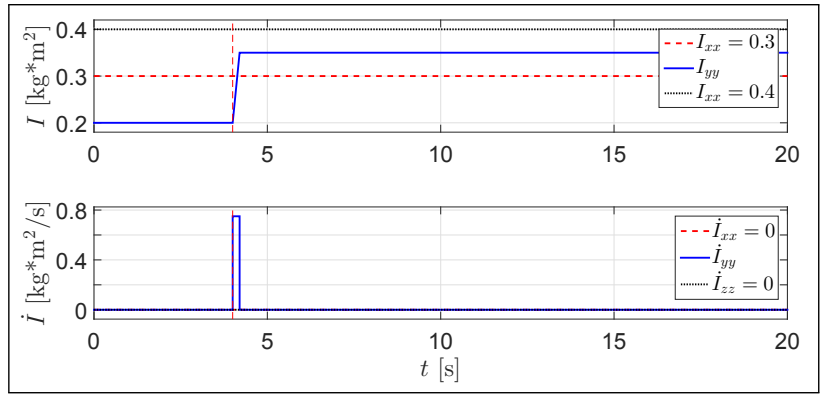

Fig. 13. Time history of the controlled manipulation with the moment of inertia $I_{y y}$ to stop flipping motion of the system.

results of the simulations, partially shown in Fig. 18.

\section{Method's Applications: Proposing New Space Missions}

It is believed, that new results of this research may have multiple applications. We would like to propose and list a few of them, which may be useful in planning new space missions: 


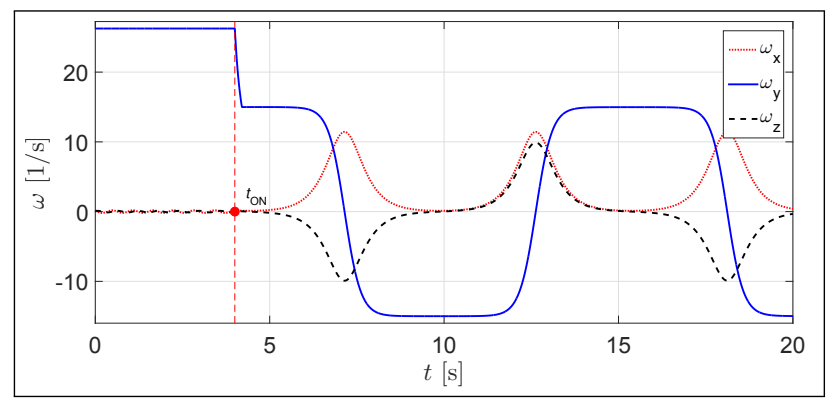

(a) Time histories of the angular velocity components of the spacecraft.

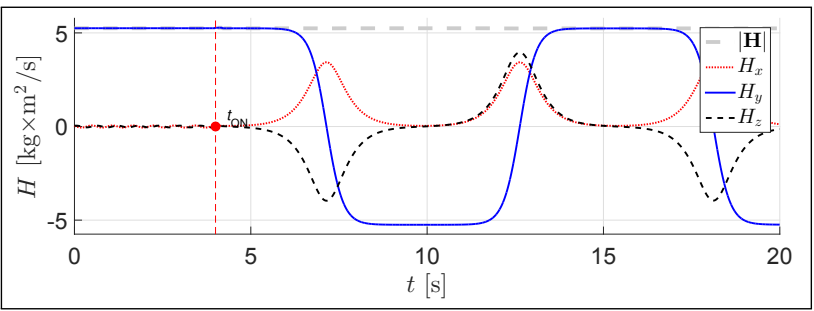

(b) Time histories of the angular momenta of the spacecraft.

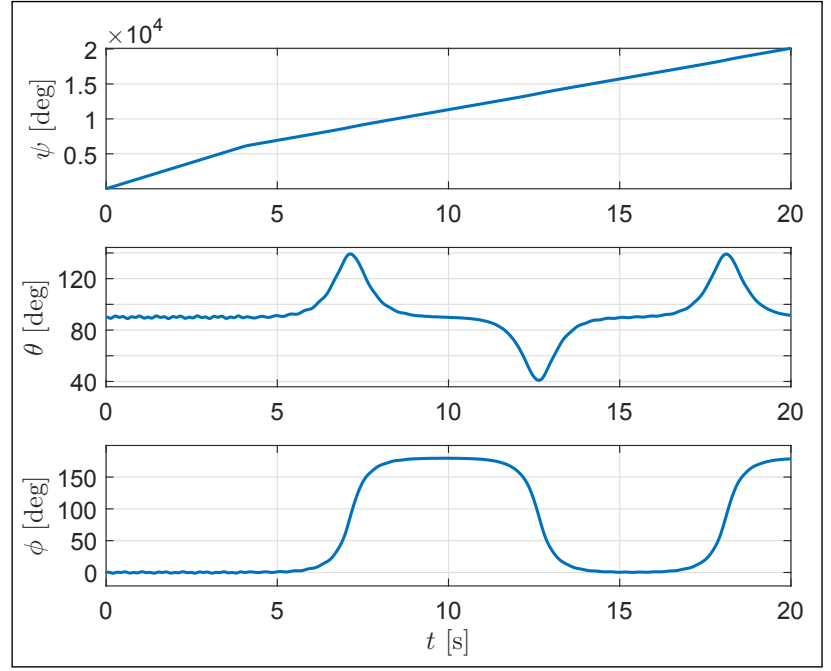

(c) Time histories of the Euler angles.

Fig. 14. Simulation results for the study Case-5: activation of the "flipping" motion of the spacecraft.

\section{Assistance in establishing formation flight:}

Eject one spacecraft in the forward direction, then, after one flip, eject another spacecraft in the opposite direction, so that the formation flying is achieved in orbit around the

Earth or other planet or celestial body (comet, asteroid).

2. Thruster direction control:

To boost (accelerate) or decelerate spacecraft velocity by

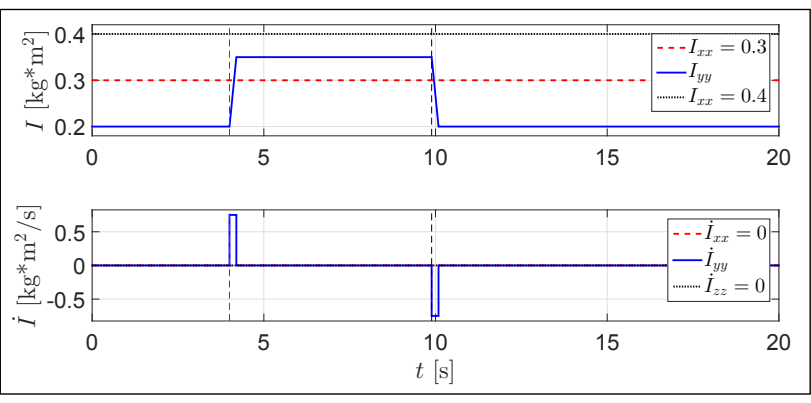

Fig. 15. Time history of the controlled manipulation with the moment of inertia $I_{y y}$ to activate flipping motion of the system and then to turn it off.

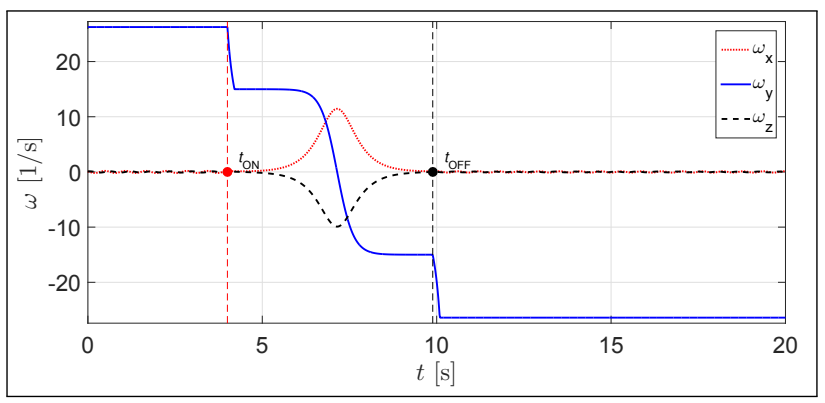

(a) Time histories of the angular velocity components of the spacecraft.

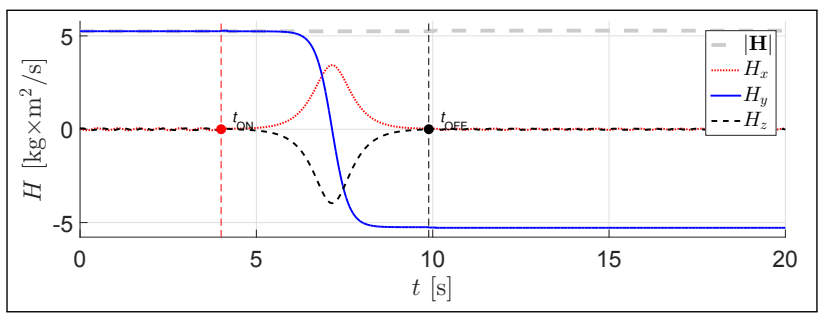

(b) Time histories of the angular momenta of the spacecraft.

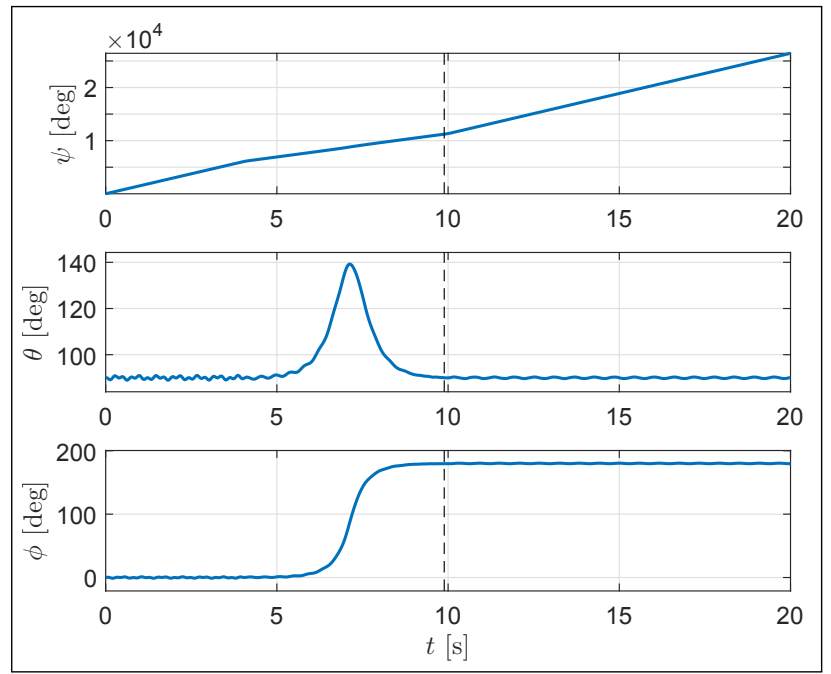

(c) Time histories of the Euler angles.

Fig. 16. Simulation results for the study Case-6: "flipping" motion is activated, one flip is performed, then "flipping" is deactivated.

only one thruster. Of course, deep space mission vehicles change its attitude to boost (escape from the Earth) and to decelerate its velocity when it enters to an orbit around the another planet. Usually another small thrusters (or reaction wheels) are used to change attitude of vehicles. In addition to this, our new proposed method could be also used for changing the attitude of the spacecraft and its following

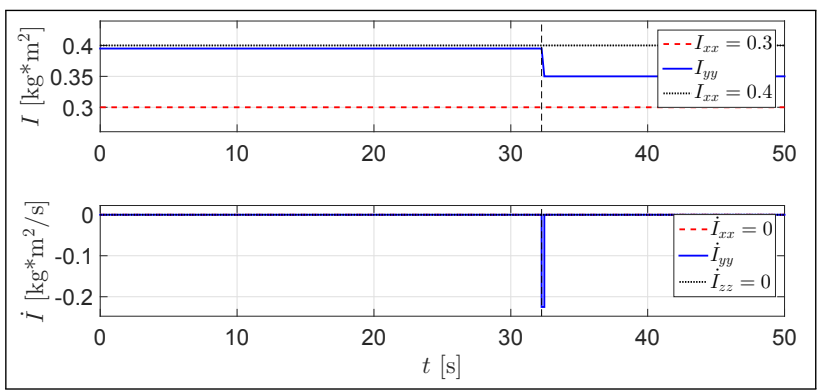

Fig. 17. Time history of the controlled manipulation with the moment of inertia $I_{y y}$ to change frequency of the flipping motion of the system. 


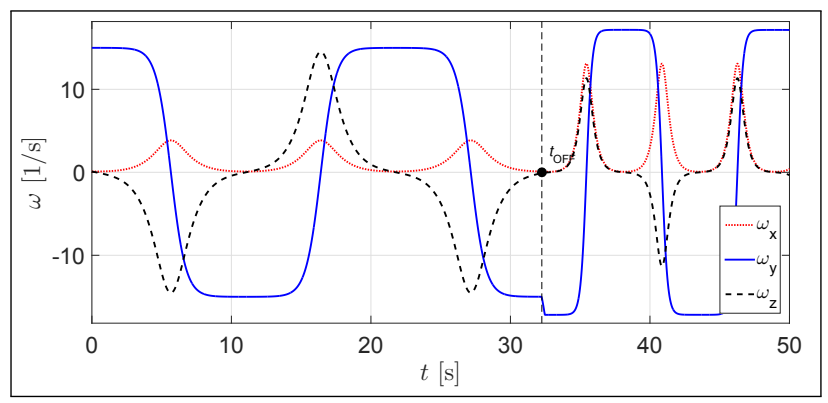

Fig. 18. Simulation results for Case-7: control of the frequency of the "flipping" motion of the spacecraft, using proposed "inertial morphing" (demonstrated with time histories of the angular velocity components).

stabilization in the fixed body frame.

3. Frequency of "flipping" motion control:

Inertial morphing can be used to control within wide range the frequency of the "flips" for the "Dzhanibekov's effect" mode. However, we showed that there is a minimum (i.e. low bounding limit) for the period of these oscillations.

\section{Main Results and Conclusions}

This paper is dedicated to the numerical simulation and analysis of the "Dzhanibekov's Effect" - non-stable "flipping" motion of the rigid body with periodic change by $180^{\circ}$ of the direction of the main axis of its rotation, always occurring when the body is provided with the main rotation about its axis with intermediate principal moment of inertia.

In this work we proposed and developed a new concept of utilizing "Dzhanibekov's Effect" for changing attitude of the spacecraft via its inertial morphing, without employing classical gyroscopes. Moreover, we proposed and tested a new method of switching OFF the "flipping" motion on the main axis of rotation by transferring motion to the stable (i.e. "non-flipping") mode. For the implementation of this transformation, we proposed two main conceptual solutions, involving changes to the system, resulting in the intermediate moment of inertia becoming the smallest or largest principal moment of inertia of the body. A conceptual model of the 6-mass model of the spacecraft enabling controllable switching OFF of the "Dzhanibekov's Effect" flipping is presented.

Furthermore, implementation of the transfer of the stable motion of the spacecraft to the unstable (i.e. "switching ON" the "flipping" mode) has also been successfully completed.

These "flipping" mode "switching ON" and "switching OFF" capabilities and their combinations have been successfully demonstrated on the representative study cases.

To achieve the main objectives in the study, we first developed a model of the tumbling spacecraft, based on the nonlinear Euler's equations of rigid body motion and successfully simulated classical "Dzhanibekov's Effects" for the rigid bodies with constant inertia properties. As main further development we enhanced the analytical and numerical models, which enabled us to simulate wide class of systems with inertial morphing (i.e. systems with variable moments of inertia). It has been proven that the conservation of angular momentum in the morphing system is observed.

It has been demonstrated that time of activation of the spacecraft controllable morphing and its duration are critical factors in the quality of stabilization and "flipping" initiation processes.

\section{References}

1) Komlev, G. Dzhanibekov's Photo from USSR Stamp, "Soyuz27", 1979. https://commons.wikimedia.org/wiki/File: USSR_stamp_Soyuz-27_1978_4k.jpg, (accessed April 9, 2017).

2) Dzhanibekov, Vladimir Aleksandrovich. Wikipedia (In Russian). https://goo.gl/ygvopE (accessed April 12, 2017).

3) Dzhanibekov, V. A.: Interview at the "Secret Signs" TV Program, https://youtu.be/dL6Pt10_gSE (published February 19, 2010 , accessed April 12, 2017).

4) "Roskosmos" Telestudio: Dzhanibekov's Effect, https://youtu . be/6ozUSgBjeaQ (published on May 15, 2012; accessed April 12, 2017).

5) Beachley, N. N.: Inversion of Spin-Stabilized Spacecraft by Mass Translation - Some Practical Aspects, J. Spacecraft, 8 (1971), pp. 1078-1080.

6) Moler, C.: Tumbling Box ODE. (Cleve's Corner: Cleve Moler on Mathematics and Computing.) Posted by Cleve Moler, August 10, 2015. http://blogs.mathworks.com/cleve/2015/08/10/ tumbling-box-ode/ (accessed June 05, 2017).

7) Murakami, H., Rios, O., Impelluso, T. J.: A Theoretical and Numerical Study of the Dzhanibekov and Tennis Racket Phenomena, Journal Applied Mechanics, 83 (2016), 111006 (10 pages). Paper No: JAM16-1017.

8) Kawano, D. T.: A Tumbling T-Handle in Space, Rose-Hulman Institute of Technology. http://rotations. berkeley.edu/?page_ id=2472 (accessed April 12, 2017).

9) Roman Vladimirovich F.: Animation of the Dzhanibekov's Effect, https://drive.google.com/drive/u/1/folders/ OB3MWZcYwMLYoRkVOMXdsdDAXSOE (accessed April 12, 2017).

10) Dzhanibekov, V. A.: Tumbling Earth: Interview at the "I Came Here to Fly" the First Educational TV Channel Program, https: //youtu . be/cy30_6m1jPc?t=901 ( accessed April 09, 2017).

11) Shkaplerov, A. and Burbank, D.: Experiments on board of the International Space Station (Expedition \#30, 2011). https: //youtu. be/LzVItPwiQyI (published February 11, 2013; accessed April 12, 2017).

12) Wakata, K.: Mysteries of Rotational Movement (Onboard of the ISS Expedition \#38, 2014), JAXA. https: //youtu. be/QhSN2 eua14I? $\mathrm{t}=309$ (published Nov 25, 2013; accessed April 06, 2017).

13) Ford, K.: Working with the Spheres Satellites Onboard of the ISS (Expedition \#34, 2013) https: //youtu.be/dsXOxcDSBLQ? $t=108$ (published Mar 13, 2013; accessed April 09, 2017).

14) Garriott, R.: Space Video Blog: Rotational Inertia, https://youtu . be/fPI-rSwAQNg (accessed April 12, 2017).

15) Plasma, B.: A T-handle tumbling in space aboard the International Space Station. https://youtu.be/1n-HMSCDYtM (published Mar 02, 2009; accessed April 12, 2017).

16) Portrait of Leonard Euler. University of Tartu collection, http:// dspace .ut . ee/handle/10062/22581?locale-attribute=en, (accessed April 12, 2017).

17) Euler, L.: "Du mouvement de rotation des corps solides autour d'un axe variable," Mémoires de lacadémie des sciences de Berlin, Vol. 14, Berlin Academy, Berlin, Germany, 1758, pp. 154-193.

18) The Euler Archive, http://eulerarchive.maa.org/ (accessed April 05, 2017).

19) Bedford, A. M. and Fowler, W.: Engineering Mechanics: Dynamics, 5th ed., Pearson, 2008. - 652 pp.

20) Landau, L. D. and Lifshitz, E. M.: Mechanics. Vol.1 (In Russian). 4th ed., Moscow, "Nauka", 1988. - 216 pp.

21) KEISAN Complete elliptic integral of the 1 st kind $K(k)$ Calculator, English version: http://keisan.casio.com/exec/system/ 1180573451, http://keisan.casio.jp/exec/system/ 1166751749 (in Japanese) (accessed June 23, 2017).

22) Trivailo, P. M. and Kojima, H.: Utilisation of the "Dzhanibekov's Effect" for the Possible Future Space Missions, Joint Conference: 31st ISTS, 26th ISSFD \& 8th NSAT, Matsuyama, Japan, ISTS-2017-d047/ISSFD-2017-047, 2017. 